\title{
Sleep Status and Low-Density Lipoprotein Particle Size in a General Japanese Female Population: The Mima Study
}

\author{
Kazuhiko Kotani ${ }^{a, b}$ Kokoro Tsuzaki ${ }^{a}$ Shinji Fujiwara ${ }^{a}$ Naoki Sakane ${ }^{a}$ \\ the Mima Study Group \\ a Division of Preventive Medicine, Clinical Research Institute, National Hospital Organization Kyoto Medical Center, \\ Kyoto, and ${ }^{\text {b }}$ Department of Clinical Laboratory Medicine, Jichi Medical University, Tochigi, Japan
}

\section{Key Words}

Atherosclerosis · Small dense low-density lipoprotein .

Sleep hygiene

\begin{abstract}
Objective: This study investigated the relationship between sleep adequacy and small dense low-density lipoprotein (sdLDL) in a Japanese population. Subjects and Methods: Clinical data, including atherosclerotic risk factors, in addition to the mean LDL particle size (LDL-PS) measured by gel electrophoresis, were evaluated in 136 community-dwelling female subjects (40-80 years old). The sleep status was selfreported and the subjects were divided into those who had inadequate sleep ( $\leq 6$ or $\geq 9$ h of sleep) and had adequate sleep (7-8 h). Results: The mean LDL-PS was significantly smaller in subjects with inadequate sleep $[n=68,26.4 \pm 0.4$ (SD) $\mathrm{nm}$ ] than in those with adequate sleep $(\mathrm{n}=68,26.6 \pm$ $0.4 \mathrm{~nm}, \mathrm{p}<0.05)$. This difference remained significant and independent after adjusting for confounders $(\beta=0.15-0.19$, $p<0.05)$. Conclusion: Sleep adequacy was associated with the presence of sdLDL, while the influence of the sleep status on LDL-PS was minor in this population. Further research on the associations among sleep, sdLDL and cardiovascular disease prevention is warranted.

Copyright $\odot 2013$ S. Karger AG, Basel
\end{abstract}

\section{KARGER}

E-Mail karger@karger.com www.karger.com/mpp

\section{(c) 2013 S. Karger AG, Basel} 1011-7571/13/0225-0510\$38.00/0

Karger

Open access

This is an Open Access article licensed under the terms of the Creative Commons Attribution-NonCommercial-NoDerivs 3.0 License (www.karger.com/OA-license), applicable to the online version of the article only. Distribution for non-commercial purposes only.

\section{Introduction}

Cardiovascular disease (CVD) is an important health problem, and the duration of sleep (short or long) predicts the occurrence of metabolic disorders [1], CVD outcomes [2] and ultimately a shortened life span [3]. Therefore, an adequate amount of sleep may help to prevent CVD events and the related mortality [3]. How an inadequate sleep status is biologically associated with CVD remains to be elucidated; however, a possible involvement of lipid/lipoprotein metabolism has been suggested [4-7].

Small dense low-density lipoprotein (sdLDL), which has a small LDL particle size (LDL-PS) with greater susceptibility to oxidation, is a risk marker for CVD [8]. Although a gene polymorphism that plays roles in human circadian rhythmicity is reportedly associated with sdLDL [6] and sleep apnea is also reported to be associated with sdLDL in nonobese people [7], there have so far been no reports regarding the potential association between general sleep status and sdLDL. The present study was aimed to investigate the relationship between sleep adequacy and the LDL-PS in a general population. 
Table 1. Clinical characteristics

\begin{tabular}{|c|c|c|c|c|}
\hline Variable & All $(n=136)$ & Adequate sleep $(n=68)$ & Inadequate sleep $(n=68)$ & $\mathrm{p}$ value \\
\hline Age, years & $68 \pm 9$ & $68 \pm 8$ & $67 \pm 10$ & 0.39 \\
\hline Body mass index & $24.4 \pm 3.0$ & $24.4 \pm 2.9$ & $24.4 \pm 3.1$ & 0.99 \\
\hline Exercise habits, $\mathrm{n}$ & $46(34)$ & $23(34)$ & $23(34)$ & 1.00 \\
\hline $\mathrm{MBP}, \mathrm{mm} \mathrm{Hg}$ & $99 \pm 13$ & $101 \pm 13$ & $97 \pm 13$ & 0.07 \\
\hline Plasma glucose, $\mathrm{mmol} / \mathrm{l}$ & $5.38 \pm 1.34$ & $5.20 \pm 1.15$ & $5.57 \pm 1.49$ & 0.11 \\
\hline Insulin, $\mu \mathrm{U} / \mathrm{ml}$ & $6.8(4.2-9.5)$ & $6.5(4.3-9.5)$ & $7.0(4.2-9.7)$ & 0.84 \\
\hline Total cholesterol, mmol/l & $5.00 \pm 0.83$ & $5.05 \pm 0.82$ & $4.96 \pm 0.85$ & 0.50 \\
\hline Triglycerides, mmol/l & $0.96(0.77-1.25)$ & $0.98(0.81-1.22)$ & $0.94(0.75-1.28)$ & 0.91 \\
\hline HDL-C, mmol/l & $1.45 \pm 0.34$ & $1.49 \pm 0.32$ & $1.40 \pm 0.35$ & 0.11 \\
\hline LDL-PS, nm & $26.5 \pm 0.4$ & $26.6 \pm 0.4$ & $26.4 \pm 0.4$ & $0.03^{*}$ \\
\hline
\end{tabular}

The data are shown as the means \pm standard deviation, medians with interquartile ranges in parentheses or subject numbers with percentages in parentheses. ${ }^{*} \mathrm{p}<0.05$ : significance level (unpaired t test: adequate sleep group vs. inadequate sleep group).

\section{Subjects and Methods}

A total of 160 community-dwelling Japanese females were consecutively recruited during general health check-ups. As rare subjects' characteristics that can influence the homogeneity of the study population, the subjects who were relatively young (under 40 years: $\mathrm{n}=4$ ), relatively old (over 80 years: $\mathrm{n}=2$ ), current smokers $(n=2)$ and abstainers from alcohol $(n=10)$, as well as those who had malnutrition conditions or thyroid disorders $(n=4)$, were also excluded. The subjects who did not report sleep duration $(n=3)$ and exercise habits $(n=3)$ were excluded. There were no subjects who had a recent history of CVD or took medications that affected sdLDL. The final study population included 136 females. The study was approved by the Ethics Committees of the National Hospital Organization Kyoto Medical Center, and each subject gave written informed consent.

Current smoking, alcohol drinking habits, sleep duration and exercise habits were self-reported. Regarding sleep adequacy, the subjects were divided into two groups based on their sleep duration on a typical day: $\leq 6$ or $\geq 9 \mathrm{~h}$ as inadequate and $7-8 \mathrm{~h}$ as adequate, based on the reported associations between sleep duration and poor health, such as CVD (short and long sleep durations were found to similarly increase the occurrence of poor health as demonstrated in a U-shaped curve) $[3,9]$. The presence of exercise habits was defined as engaging in structured exercise of $>30$ min twice a week. All biochemical data were obtained after an overnight fast. In addition to the body mass index (BMI) and mean blood pressure (MBP), the serum total cholesterol, triglycerides, high-density lipoprotein cholesterol (HDL-C) and plasma glucose concentrations were enzymatically measured (Kyowa Medics Corp., Tokyo, Japan). The plasma insulin level was measured by an ELISA system (Tosoh Co. Inc., Tokyo, Japan) and the homeostasis model assessment of insulin resistance (HOMA-IR) was calculated as follows: fasting glucose $\times$ insulin/22.5. The mean LDL-PS was simultaneously measured with a high-resolution, nongradient polyacrylamide gel electrophoresis system (the Lipoprint system; Quantimetrix, Redondo Beach, Calif., USA) that was validated using the gold standard method of nuclear magnetic resonance spectroscopy [6].
The data are expressed as the means \pm standard deviation or the medians plus interquartile range (for triglyceride, insulin and HOMA-IR that had the skewed distributions of their values). The differences between groups were compared using the unpaired $t$ tests or one-way ANOVA with Tukey tests. A general linear or multiple regression model for LDL-PS (as a dependent variable) was utilized to examine the influence of sleep adequacy (as a fixed variable) with adjustments for all measured variables (the SPSS ${ }^{\circledR}$ version 16; SPSS Inc., Chicago, Ill., USA). The triglyceride, insulin and HOMA-IR values were log-transformed in all the analyses. A $p$ value $<0.05$ was considered to be statistically significant.

\section{Results}

Of 59 subjects with $\leq 6 \mathrm{~h}$ of sleep, the mean age was 66 \pm 10 years and LDL-PS levels were $26.4 \pm 0.4 \mathrm{~nm}$; as well, the mean age was $73 \pm 6$ years and LDL-PS levels were 26.2 $\pm 0.5 \mathrm{~nm}$ in 9 subjects with $\geq 9 \mathrm{~h}$ of sleep. Of 68 subjects with adequate sleep, the mean age was $68 \pm 8$ years and LDL-PS levels were $26.6 \pm 0.4 \mathrm{~nm}$. A significant difference was observed in LDL-PS among these subject groups $(\mathrm{p}=$ 0.03 ), and in particular, the LDL-PS level of those with $\geq 9$ h was significantly smaller than that of those with adequate sleep $(\mathrm{p}=0.04)$. There was no significant difference in age among these groups. As shown in table 1, the subjects reporting inadequate sleep exhibited significantly smaller LDL-PS than those reporting adequate sleep, while nonsignificant differences in the other variables were observed between the subjects with inadequate and adequate sleep. The LDL-PS levels remained significantly and independently different, even after adjusting for the other measured variables: model 1 was adjusted for age, BMI and exercise habits $(\beta=0.19, \mathrm{~F}=5.00, \mathrm{p}=0.03)$, model 2 was 
adjusted for age, BMI, MBP, total cholesterol, triglycerides, HDL-C, glucose, insulin and exercise habits $(\beta=0.15$, $\mathrm{F}=4.58, \mathrm{p}=0.03$ ), and model 3 was adjusted for age, BMI, MBP, total cholesterol, triglycerides, HDL-C, HOMA-IR and exercise habits $(\beta=0.15, \mathrm{~F}=4.78, \mathrm{p}=0.03)$.

\section{Discussion}

The present study found that female subjects reporting inadequate sleep had significantly smaller LDL-PS, independently of confounders, than those reporting adequate sleep. The influence of the sleep status on LDL-PS appeared to be minor. Therefore, the clinical relevance of the difference in LDL-PS between the two subject groups should be confirmed in further studies, but we cannot ignore the fact that even such a minor difference in the LDLPS level (i.e. $0.2 \mathrm{~nm}$ ) makes it possible to clearly distinguish the diabetic and nondiabetic pathophysiology, as reported in an earlier study [10]. The present study result could also support a recent report finding that shows sleep apnea to be associated with the existence of sdLDL [7]. These findings suggest that an inadequate sleep status may be associated with an atherogenic lipoprotein profile, such as sdLDL, and in turn the occurrence of CVD, as a result of altered lipoprotein metabolism. In addition, the present study showed the possibility that the subjects with a long sleep duration of $\geq 9$ h could have a smaller LDL-PS than those with adequate sleep, but this study included only a small number of subjects who demonstrated a long sleep duration (this phenomenon of a small percentage of longer sleepers is common in developed countries [3]); therefore, the result merits further investigation.

While the precise mechanism(s) underlying the association between sleep adequacy and sdLDL remain unknown, there are several possible explanations. Sleep problems and related hypoxia can inhibit the clearance of triglyceride-rich lipoproteins and decrease the lipoprotein lipase activities [5], which promotes the formation of sdLDL particles [8]. The presence of sdLDL can also be determined partly by circadian rhythmicity-related genetic components [6].

The present study has several limitations. First, the study included a relatively small number of subjects, and all of the subjects were females. The study had a crosssectional design, which does not completely allow for a determination of the causality of the results. No definitive conclusions can be made regarding whether adequate sleep can help to prevent CVD events until additional intervention studies can be carried out. The sleep status was subjectively assessed by self-reports (in contrast to a report using the apnea hypopnea index [7]), and sleep patterns (e.g. time awake and asleep) as well as sleep problemrelated symptoms (e.g. daytime sleepiness) were not evaluated. Exercise habits did not evaluate the amount and duration of exercise, either. A future study will address these issues.

\section{Conclusion}

The Japanese female subjects with inadequate sleep showed a significantly smaller LDL-PS than those with adequate sleep, thus suggesting that inadequate sleep may be associated with the presence of sdLDL, a CVD risk marker. More studies are necessary to confirm the observed relationship and the clinical meaning of these findings.

\section{Acknowledgements}

This research was supported in part by a Grant-in-Aid for the Foundation for the Development of the Community, Japan.

\section{References}

$>1$ Choi KM, Lee JS, Park HS, et al: Relationship between sleep duration and the metabolic syndrome: Korean National Health and Nutrition Survey 2001. Int J Obes 2008;32:1091-1097.

$>2$ Cappuccio FP, Cooper D, D'Elia L, et al: Sleep duration predicts cardiovascular outcomes: a systematic review and meta-analysis of prospective studies. Eur Heart J 2011;32:14841492.

$>3$ Luyster FS, Strollo PJ Jr, Zee PC, et al: Boards of Directors of the American Academy of Sleep Medicine and the Sleep Research Society: Sleep: a health imperative. Sleep 2012;35:727-734.

$\checkmark 4$ Rudic RD, McNamara P, Curtis AM, et al: BMAL1 and CLOCK, two essential com- ponents of the circadian clock, are involved in glucose homeostasis. PLoS Biol 2004; 2:e377.

5 Drager LF, Li J, Shin MK, et al: Intermittent hypoxia inhibits clearance of triglyceride-rich lipoproteins and inactivates adipose lipoprotein lipase in a mouse model of sleep apnoea. Eur Heart J 2012;33:783-790.

6 Tsuzaki K, Kotani K, Sano Y, et al: The association of the Clock 3111 T/C SNP with lipids and lipoproteins including small dense lowdensity lipoprotein: results from the Mima study. BMC Med Genet 2010;11:150.

$\checkmark 7$ Luyster FS, Kip KE, Drumheller OJ, et al: Sleep apnea is related to the atherogenic phe- notype, lipoprotein subclass B. J Clin Sleep Med 2012;8:155-161.

$>8$ Packard CJ: Small dense low-density lipoprotein and its role as an independent predictor of cardiovascular disease. Curr Opin Lipidol 2006;17:412-417.

9 Amagai Y, Ishikawa S, Gotoh T, et al: Sleep duration and mortality in Japan: the Jichi Medical School Cohort Study. J Epidemiol 2004; 14:124-128.

10 Suh S, Park HD, Kim SW, et al: Smaller mean LDL particle size and higher proportion of small dense LDL in Korean type 2 diabetic patients. Diabetes Metab J 2011;35: 536-542. 